\title{
Effects of MicroRNA-206 on Osteosarcoma Cell Proliferation, Apoptosis, Migration and Invasion by Targeting ANXA2 Through the AKT Signaling Pathway
}

\author{
Bao-Long Pan ${ }^{\mathrm{a}} \quad$ Zong-Wu Tong ${ }^{\mathrm{b}}$ Ling Wuc $\mathrm{Li}^{\mathrm{C}}$ Pan ${ }^{\mathrm{a}} \quad$ Jun-E Lia \\ You-Guang Huang ${ }^{d}$ Shu-De Lie Shi-Xun Dua Xu-Dong $\mathrm{Li}^{\mathrm{a}}$
}

aDepartment of Laboratory, People's Hospital of Yuxi City, Yuxi, 'bepartment of nephrology, People's Hospital of Yuxi City, Yuxi, 'Department of Quality Control, Central blood station of Yuxi city, Yuxi, 'Tumor Markers Research Center, Tumor Institute of Yunnan Province, The Third Affiliated Hospital of Kunming Medical University, Kunming, eDepartment of Biochemistry and Molecular Biology, School of Basic Medical Science, Kunming Medical University, Kunming, P.R. China 